\title{
Next-generation sequencing of patients with congenital anosmia
}

\author{
Anna Alkelai ${ }^{1,2} \cdot$ Tsviya Olender $^{1} \cdot$ Catherine Dode $^{3,4} \cdot$ Sagit Shushan $^{5,6} \cdot$ Pavel Tatarskyy $^{1} \cdot$ Edna Furman-Haran $^{7}$. \\ Valery Boyko $^{1} \cdot$ Ruth Gross-Isseroff $^{1} \cdot$ Matthew Halvorsen $^{2} \cdot$ Lior Greenbaum $^{8,9} \cdot$ Roni Milgrom ${ }^{1}$. \\ Kazuya Yamada $^{10} \cdot$ Ayumi Haneishi $^{11} \cdot$ Ilan Blau $^{12} \cdot$ Doron Lancet $^{1}$
}

Received: 22 March 2017 / Revised: 10 August 2017 / Accepted: 9 September 2017 / Published online: 13 November 2017

(c) European Society of Human Genetics 2017

\begin{abstract}
We performed whole exome or genome sequencing in eight multiply affected families with ostensibly isolated congenital anosmia. Hypothesis-free analyses based on the assumption of fully penetrant recessive/dominant/X-linked models obtained no strong single candidate variant in any of these families. In total, these eight families showed 548 rare segregating variants that were predicted to be damaging, in 510 genes. Three Kallmann syndrome genes (FGFR1, SEMA3A, and CHD7) were identified. We performed permutation-based analysis to test for overall enrichment of these 510 genes carrying these 548 variants with genes mutated in Kallmann syndrome and with a control set of genes mutated in hypogonadotrophic hypogonadism without anosmia. The variants were found to be enriched for Kallmann syndrome genes ( 3 observed vs. 0.398 expected, $p=0.007)$, but not for the second set of genes. Among these three variants, two have been already reported in genes related to syndromic anosmia (FGFR1 (p.(R250W)), CHD7 (p.(L2806V))) and one was novel (SEMA3A (p.(T717I))). To replicate these findings, we performed targeted sequencing of 16 genes involved in Kallmann syndrome and hypogonadotrophic hypogonadism in 29 additional families, mostly singletons. This yielded an additional 6 variants in 5 Kallmann syndrome genes (PROKR2, SEMA3A, CHD7, PROK2, ANOS1), two of them already reported to cause Kallmann syndrome. In all, our study suggests involvement of 6 syndromic Kallmann genes in isolated anosmia. Further, we report a yet unreported appearance of di-genic inheritance in a family with congenital isolated anosmia. These results are consistent with a complex molecular basis of congenital anosmia.
\end{abstract}

\section{Introduction}

Congenital anosmia is an uncommon condition that is defined as absence of sense of smell from birth [1,2]. Complete loss of olfactory sensory function is rather frequent and affects $5 \%$ of the general population [1]. However, only 1 in 10,000 have a complete loss of olfaction

(https://doi.org/10.1038/s41431-017-0014-1) contains supplementary material, which is available to authorized users.

Anna Alkelai

aa3857@cumc.columbia.edu

1 Deptment of Molecular Genetics, Weizmann Institute of Science, Rehovot, Israel

2 Institute for Genomic Medicine, Columbia University Medical Center, New York, NY, USA

3 Hôpital Cochin, AP-HP, Université Paris-Descartes, Paris 75004, France

4 Laboratoire de Biochimie et Génétique Moléculaire, Hôpital Cochin, Paris, France

5 Department of Neurobiology, Weizmann Institute of Science, Rehovot, Israel

6 Department of Otolaryngology-Head and Neck Surgery, Edith

Wolfson Medical Center, Holon 58100, Israel

7 Life Sciences Core Facilities, Weizmann Institute of Science, Rehovot, Israel

8 The Danek Gertner Institute of Human Genetics, Sheba Medical Center, Tel Hashomer, Ramat Gan, Israel

9 The Joseph Sagol Neuroscience Center, Sheba Medical Center, Tel Hashomer, Ramat Gan, Israel

10 Matsumoto University Graduate School of Health Science, Nagano, Japan

11 Department of Health and Nutritional Science, Faculty of Human Health Science, Matsumoto University, Nagano, Japan

12 Department of Otolaryngology, Meir Medical Center, Kfar Saba, Israel 
from birth [2]. Congenital anosmia could be isolated, without other symptoms, or syndromic [1]. Syndromes in which congenital anosmia presents as one of the traits include Kallmann syndrome (OMIM \#308700) [3], congenital insensitivity to pain (OMIM \#243000) [4], CHARGE syndrome (OMIM \# 214800), Bardet -Biedl syndrome (OMIM \#209900) [5], Leber congenital amaurosis (OMIM \#611755) [6], Refsum disease (OMIM \#266500) [7] and additional syndromes. Broader list of syndromes, candidate genes and links to the detailed phenotypic information can be found in Supplementary table 1 .

While the molecular mechanisms underlying olfaction have been studied in considerable detail, the molecular genetic basis of isolated congenital anosmia is only scantly addressed [1]. Specific familial cases with various types of inheritance were described [1, 8, 9]. Only few variants underlying isolated congenital anosmia have been reported so far, in the genes PROKR2, PROK2 [10], CNGA2 [8], and TENM1 [11].

The most extensively investigated syndromic form of congenital anosmia is Kallmann syndrome, which is characterized by hypogonadotrophic hypogonadism with anosmia or hyposmia. Previous studies highlighted the variable phenotypic expression of this disorder [1, 12]. The degree of the hypogonadism and smell deficiency can vary significantly, not only between unrelated patients, but also within isolated congenital anosmia families [13, 14]. Thus, in some Kallmann syndrome families, some individuals with isolated anosmia are reported [15]. Therefore, it may be possible that the genetic architecture of these two conditions (isolated anosmia and Kallman syndrome) is partially overlapping, and several genes might be involved in both. Few genes were reported as involved in Kallmann syndrome, among them are ANOS1 (also named KAL1), FGFR1, PROKR2, PROK2, CHD7, and SEMA3A [16, 17]. Previous studies showed that Kallmann syndrome may be inherited in X-linked recessive, autosomal dominant or autosomal recessive modes of inheritances [16-19]. In addition, digenic/oligogenic mode of inheritance has been also described [16-19].

To better understand the genetic basis of isolated congenital anosmia, we recruited cohort of 107 families affected with isolated congenital anosmia (without a reported infertility or any alternative medical explanation for the general anosmia) and studied in some detail 37 of them; including 8 families that were multiply affected/ consanguineous. We used hypothesis-free next-generation sequencing (whole exome sequencing and in some cases whole genome sequencing) to study the genetic variations in 32 selected individuals coming from these 8 families. Furthermore, we applied a hypothesis driven approach to investigate enrichment of genes known to be mutated in Kallmann syndrome among the list of variants segregating in our families. We further validated our results by utilizing gene panel sequencing to another 29 families/singeltons. The results suggested that ostensibly isolated congenital anosmia may be caused by variations in Kallmann syndrome genes more often than expected, and can also be inherited in di-genic manner. This implies that isolated congenital anosmia is more genetically complex than previously thought.

\section{Materials and methods}

\section{Clinical methods and cohorts}

The study was conducted with the approval of the Institutional Review Board for human experiments in the Meir Hospital, Kfar Saba, Israel. The informed consent was signed by all participants. Isolated congenital anosmia cohort, including 17 families with more than one isolated congenital anosmia affected individual, was recruited and phenotyped as previously described $[2,11]$. Test criteria for isolated congenital anosmia are (a) reported complete lack of the sense of smell for an entire lifespan; (b) null performance in a three-way forced choice olfactory sensitivity tests for the standard odorants isoamyl-acetate and eugenol; (c) the lack of any alternative medical explanation for the general anosmia including head trauma, sinonasal disease, failure to complete puberty or infertility (self-reported). Similar to other previously published studies [2, 8], our diagnosis was based on the exclusion of alternative underlying causes, but we did not perform broader clinical evaluations to exclude biochemically hipogonadism, by measuring plasma level of luteinizing hormone, follicle-stimulating hormone, testosterone, and estradiol, which could strengthen our assertion. Therefore, throughout this paper we use the term "ostensibly isolated congenital anosmia".

\section{Sequencing and bioinformatics}

Genomic DNA was extracted from whole blood or saliva (Oragene•DNA (OG-500), DNA Genotek, Ontario, Canada) by standard methods. Illumina HiSeq 2500 next-generation sequencing was performed. Agilent SureSelect Human All Exon Kit (Agilent, Santa Clara, CA) or Illumina TrueSeq Exome Enrichment Kit (Illumina, San Diego, CA) were used for whole exome capture. The capture kit type was consistent within a given family. In selected cases from families where the genetic transmission appears to be most consistent with single-gene inheritance, whole genome sequencing was carried out (A001-3, A210-1, and 210-2) to exclude intergenic disease variants and copy number variations (CNVs).

For variant calling we used an automatic pipeline that we developed in our laboratory. The pipeline was based on 
BWA [20] for sequence alignment, Samtools [21] and GATK [22] for variant calling and ANOVAR [23] software for variant annotation. The variants frequency in the general population was assessed by screening the 1000 genomes project, the Exome Aggregation Consortium (ExAC) and NHLBI exome sequencing database, Complete Genomics whole genome data and our own exome database with $\sim 408$ Israeli exomes. Functional prediction of non-synonymous variants was assessed by screening the dbNSF database [24] which contained predictions from 8 algorithms. When applying the dominant model with partial penetrance, we took only the Polyphen 2 predictions into consideration. CNVs were assessed by using of ERDS [25] and CoNIFER software [26]. The IBD (identity by descent) check was done by using Plink [27] and KING [28] softwares. Gene prioritization was also assisted by our published olfactory candidate gene database [29] and a novel tool that was developed by our group, VarElect [30].

Our initial assumption was that completely penetrant genotypes would explain the ostensibly isolated congenital anosmia in each single patient. We expected that the variants appear in all anosmia affected individuals per family, while appear in very low frequency $(<3 \%$ for recessive and $<1 \%$ for dominant model) in controls. Each family was studied under implementation of several genetic models: recessive (in which a shared homozygous variant is expected or compound heterozygous), X-linked recessive, dominant and dominant with partial penetrance. As a part of the effort to identify the disease causing variants, we also performed whole genome SNP genotyping (Affymetrix human SNP 6.0 array) for A001 and A039 families, and a whole genome sequencing of selected individuals coming from families A001 (1) and A210 (1 and 2). The results of the SNP array were used to identify regions with CNVs and candidate linkage genomic intervals. These results, together with the whole genome sequencing, were integrated with the exome sequencing data. All the candidate variants were validated by Sanger sequencing.

\section{Gene set enrichment analysis}

We checked whether genes harboring segregating variants within them were enriched for two sets of major genes mutated in Kallmann syndrome (ANOS1, AXL, CHD7, FEZF1, FGF17, FGFR1, HESX1, HS6ST1, IL17RD, NSMF, PROK2, PROKR2, SEMA3A, SEMA7A, SOX10,WDR11, FGF8) and hypogonadotrophic hypogonadism without anosmia (DMXL2, GNRH1, GNRHR, KISS1, KISS1R, LEP, LEPR, NROB1, OTUD4, PCSK1, PNPLA6, RNF216, TAC3, TACR3) taken from Kim at al. 2015 [16]. The detailed exome coverage of the Kallmann gene set can be found in a Supplementary table 2. An internally developed workflow was used to test the statistical significance of hypothesis as previously described [31].
We randomly selected an equal number of protein coding genes captured by our exome study, which are matched with the original set of genes in regards to total length, sequencing coverage and intolerance (RVIS score [32]), and then counted the genes that overlap with the input gene set. The $p$-value was calculated as the "rank" of our observed gene overlap in the full distribution of overlaps from 10,000 samplings of gene sets. This approach was previously successfully used to study another complex disease [31].

\section{Magnetic resonance imaging (MRI) protocol and measurements}

The MRI protocol was approved by the ethical committee of Wolfson Medical Center, Holon. All anosmic patients from the A001 family and unrelated control males were examined on a 3 Tesla whole body MRI scanner: MAGNETOM Trio, Tim System (Siemens, Erlangen, Germany), equipped with a 32-channels head coil. Coronal slices (35 slices), covering the olfactory bulb and olfactory sulcus regions, were acquired with $2 \mathrm{D}$ T2-weighted turbo spin echo pulse sequence, with slice thickness of $1.6 \mathrm{~mm}$ (no gap) and $0.4 \times 0.4 \mathrm{~mm}$ in-plane resolution, echo time of 85 $\mathrm{ms}$, repetition time of $7000 \mathrm{~ms}$, and 2 averages. For the image reconstruction we used Siemens's tool that allows 3D reconstruction and interactive move through $3 \mathrm{D}$ volumes at arbitrary orientation (3D MPR-3D multiplanar reconstruction (MPR) tool (Syngo MR, Siemens)).

The olfactory bulb volume and olfactory sulcus depth were measured according to the standard method described by Rombaux and colleagues [33, 34]. The olfactory bulb volume was computed by plannimertic manual countering the olfactory bulb surface in each coronal slice $\left(\mathrm{mm}^{2}\right)$, following by multiplying the obtained sum by slice thickness (1.6 mm with no inter-slices gap) in order to calculate olfactory bulb volume $\left(\mathrm{mm}^{3}\right)$.

The olfactory sulcus depth was measured at the level of the PPTE (plane of the posterior tangent through the eyeballs) which transverse the anterior-mid segment of the olfactory bulb. First we drew a virtual line tangent to the inferior border of the orbital and rectus gyri, than we marked a perpendicular line connecting the above virtual line and the deepest part of the olfactory sulcus, line that represents the olfactory sulcus depth. To compare the regions of interest size between cases and controls, we used a $t$-test.

\section{Kallmann syndrome and hypogonadotrophic hypogonadism gene panel sequencing}

For replication analysis in additional 29 patients we applied next-generation sequencing of a gene panel available in the Laboratoire de Biochimie et Génétique Moléculaire in Cochin Hospital, France. Variants were sought in the 
coding exons and flanking splice sites of the 16 following genes: CHD7, KAL1, FGFR1, FGF8, PROKR2, PROK2, WDR11, HS6ST1, SEMA3A, SOX10, GNRHR, GNRH1, TACR3, TAC3, KISSIR, and KISS1 as previously described [35]. The targeted sequencing covered $98 \%$ of the target regions. The following exons were not completely covered by NGS sequencing: exon 1 ANOS1, exon 1 PROK2, exon 5 KISS1R, exon 11 SEMA3A, exon 17 FGFRI. All the exons that were not fully covered by the targeted sequencing were sequenced using Sanger method. We did not use the minor allele frequency as filter for candidate variants found by the targeted sequencing, since some variants can be frequents and pathogenic (such as p.V435I in SEMA3A, that functional tests demonstrated its pathogenicity) [36].

\section{Results}

Rare variants were sought in eight multiply affected families with ostensibly isolated congenital anosmia, of which six were consanguineous (Fig. 1a, b). These were analyzed with integration of data from whole exome sequencing, whole genome SNP genotyping, and whole genome sequencing. We first performed analyses based on the assumption of fully penetrant recessive/dominant/X-linked models and obtained no strong candidates in any of the families.

We subsequently applied a model of dominant inheritance with incomplete penetrance (all anosmic individuals within the family carry the variant, as well as some of the non-affected family members). After filtration by control allele frequency $<0.01$ that is more appropriate for dominant model with partial penetrance, we found 548 rare segregating, mostly heterozygous, functional variants (frameshift, splicing, stop gain and nonsynonymous variants that were predicted to be damaging by Polyphen2) in 510 genes. None of these variants was found in genes known to cause isolated anosmia like PROKR2, PROK2 [10], CNGA2 [8] and TENMI [11]. However, three Kallmann syndrome genes (FGFR1, SEMA3A and CHD7) out of the 510 genes were identified.

In order to study the possibility of genetic overlap between isolated anosmia and Kallman syndrome, we performed permutation-based analysis to test for overall enrichment with two sets of genes (see complete gene list above): Kallmann syndrome genes $(n=17)$ and genes that cause hypogonadotrophic hypogonadism without anosmia $(n=14)$ as a negative control gene set [16]. The $548 \mathrm{seg}$ regating variants in our families were found to be enriched for Kallmann syndrome genes (3 observed vs. 0.398 expected, $p=0.007)$. No enrichment was found with hypogonadotrophic hypogonadism genes, as expected. Among these three variants, one has been already reported to cause Kallmann (ENST00000447712: c.748C > T, p.
(R250W)) [37-39], the second is located within a gene that causes CHARGE syndrome, CHD7 (ENST00000307121: c. $8416 \mathrm{C}>\mathrm{G}$, p.(L2806V)) $[40,41])$ and the third is a novel variant found in SEMA3A gene (ENST00000265362: c. $2150 \mathrm{C}>\mathrm{Tp} .(\mathrm{T} 717 \mathrm{I})$ ) (Table 1).

Two of the variants were found in Family A001. This family of Iraqi/Ashkenazi Jewish origin underwent a more thorough analysis than others, including work published previously [2]. First, an X-linked mode of transmission was tested as it has 3 anosmic sons and a self-reported anosmic grandfather (deceased) [2]. Using SNP array we identified a single $695 \mathrm{~kb}$ interval on chromosome $\mathrm{X}$ (chrX: $22,205,937-22,900,593)$ shared by all anosmic male siblings. Whole genome sequencing, which was later applied to individual A001-3 and fully covered this region, did not identify any candidate rare variant in this interval, including non-coding variants and copy number variations. We subsequently implemented whole exome sequencing to the three anosmic individuals, their healthy parents and uncle (Fig. 1). We did not identify any strong candidate rare homozygote, compound heterozygous or hemizygous variants. By applying hypothesis driven approach, two heterozygous missense variants in the genes SEMA3A and FGFRl emerged as the only candidates with known involvement in anosmia. The FGFRl variant (ENST00000447712:c. $748 \mathrm{C}>\mathrm{T}$; p.(R250W)) was from paternal origin and the SEMA3A variant (ENST00000265362:c. 2150 C > T; p.(T717I)) from maternal origin (Fig. 2). This is consistent with a digenic mode of inheritance (Fig. 2). As mentioned above, both FGFRI and SEMA3A are implicated in Kallmann's syndrome, including in di-genic inheritance [14, 36, 42, 43].

Kallmann syndrome was shown to be associated with a variety of non-reproductive developmental abnormalities including abnormal peripheral olfactory system development (olfactory nerve and olfactory bulb) [44, 45]. To examine the involvement of these brain structures in our family members, we performed MRI scan of the three anosmic brothers from A001 family and four control individuals without anosmia. The result demonstrated significant smaller bilateral olfactory bulb size in anosmic siblings (Fig. 3, Table 2) $(p=0.0019)$ compared to controls, supporting degree of agenesis of the bulb. This particular family was carefully clinically examined and questioned by an experienced physician, to exclude additional symptoms that may suggest hypogonadism, in all anosmic patients. Moreover, all of the anosmic individuals reported normal puberty and fertility. However, blood tests for sex hormones measurements should still be done in all affected with anosmia siblings to completely exclude the existence of hypogonadism.

The third variant was found in family A027. A missense (p.(L2806V)) CHD7 variant (ENST00000307121; c.8416C 
$>$ G) was found in both anosmic siblings in this family. The same variant was previously reported as a variant of unknown significance in a patient with suspected diagnosis of CHARGE syndrome [40, 41].

a

$\star$ WES or WGS

A001, FGFR1 and SEMA3A mutations
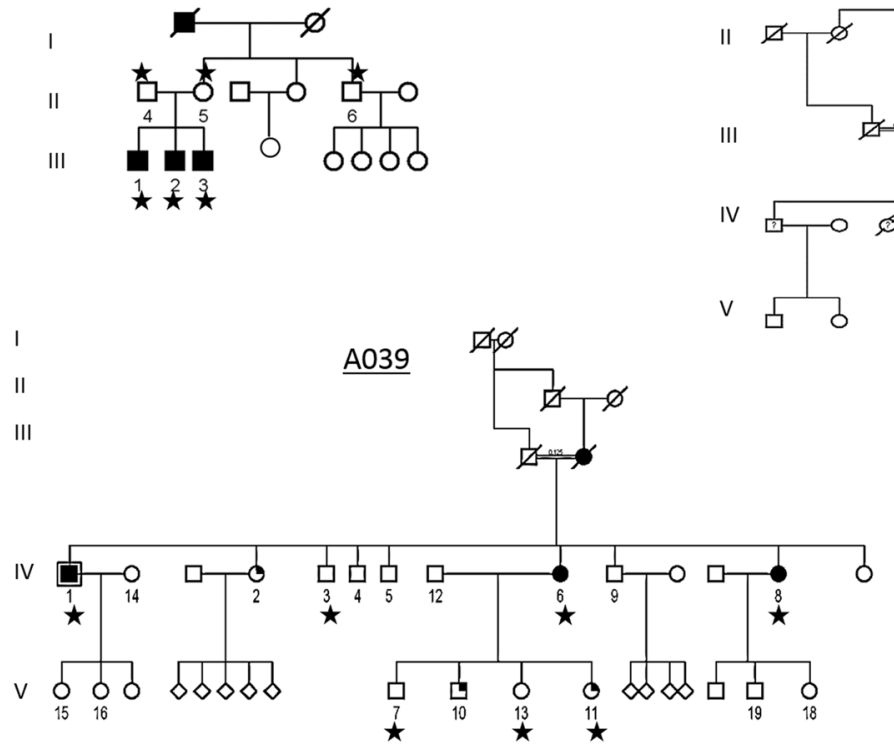

A027, CHD7 mutation

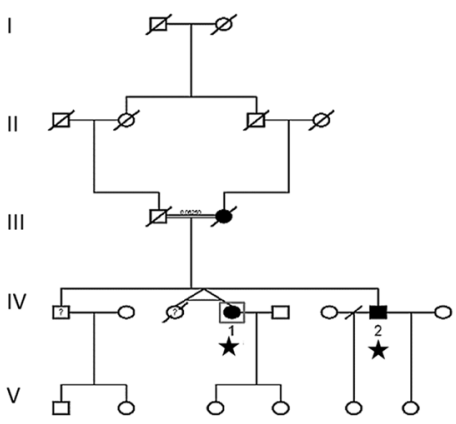

A071, SEMA3A mutation

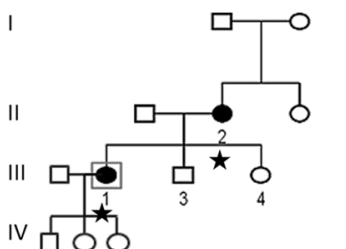

b

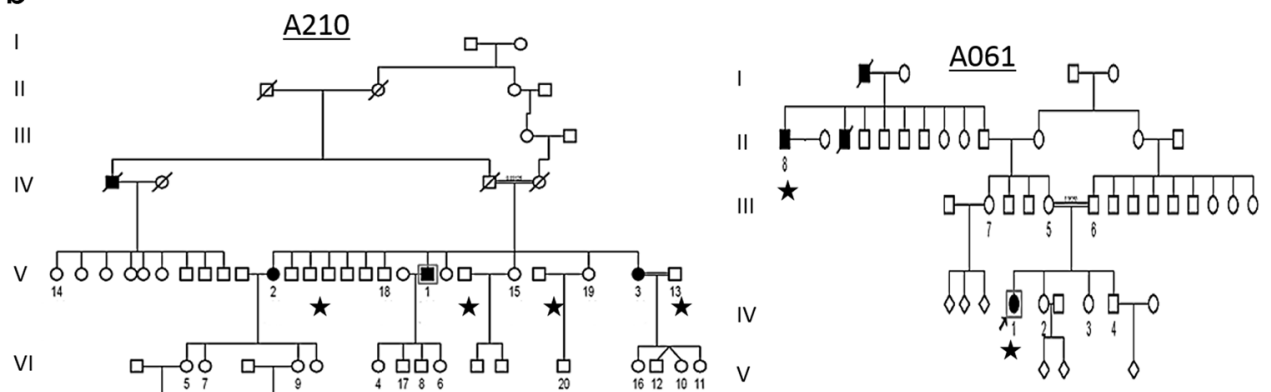

To replicate our enrichment results, we used targeted sequencing of coding regions of 16 Kallmann syndrome and hypogonadotrophic hypogonadism genes to screen representative patients from additional 29 un-deciphered isolated 


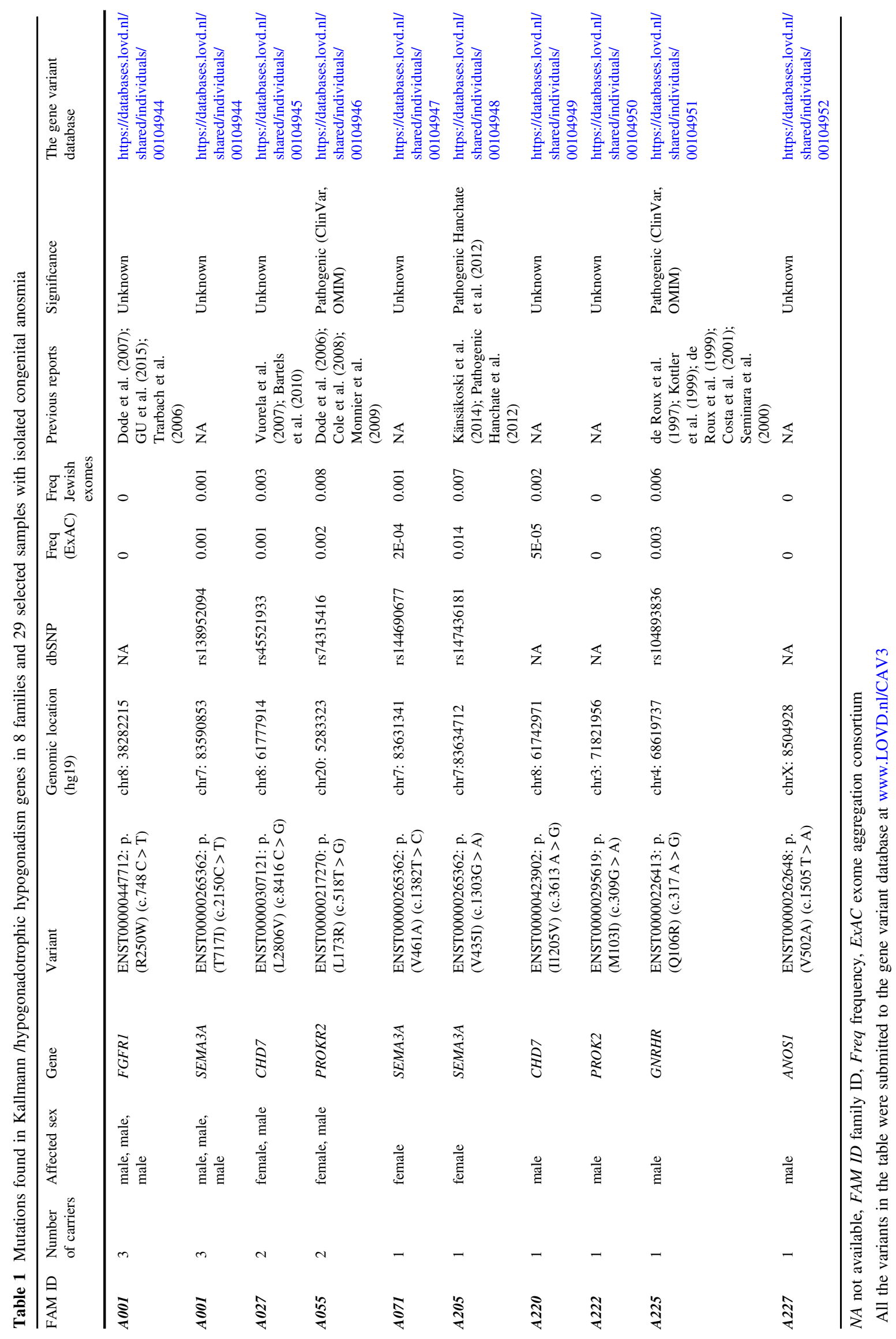




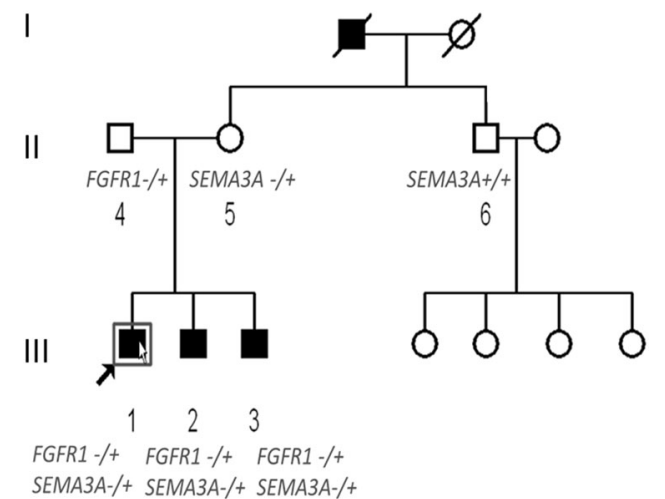

Fig. 2 Segregation analysis suggests a di-genic mode of inheritance in A001 family

\section{a)}

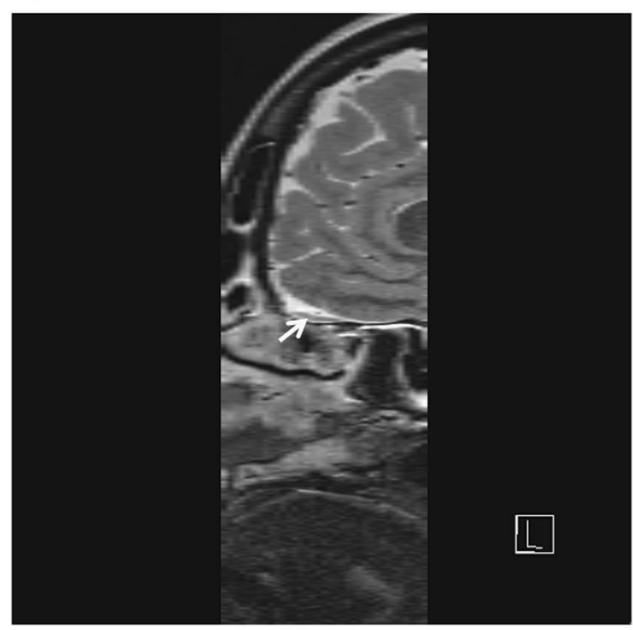

b)

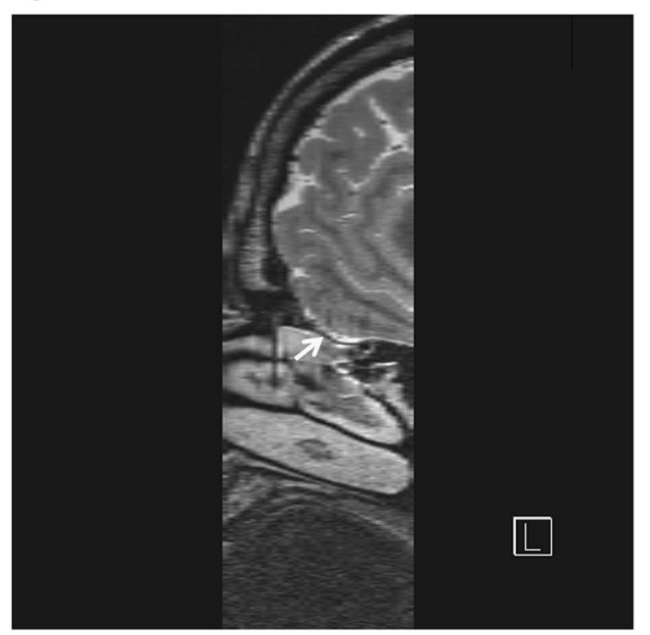

Fig. 3 a MRI scan of the control subject (N3). b MRI scan of one of the affected brothers (patient III-2 in family A001): bilateral olfactory bulb agenesis is shown in the patient. The right panel shows the raw congenital anosmia families in our cohort. In seven families (24\%) we identified missense heterozygous rare variants in Kallmann syndrome/hypogonadotrophic hypogonadism genes. In family A055 (Fig. 4) a PROKR2 variant (ENST00000217270: c.518T > G, p.(L173R)) showed perfect segregation via RFLP (restriction fragment length polymorphism) analysis in one anosmic and two heathy siblings. Three of the variants discovered were previously found in other reported Kallmann syndrome/hypogonadotrophic hypogonadism cases (SEMA3A (ENST00000265362: c.1303G > A, p.(V435I)), PROKR2 (ENST00000217270: c.518T > G, p.(L173R)), GNRHR (ENST00000226413: c.317A $>$ G, p.(Q106R))), and their functional effect was further validated (Table 1). Of particular interest is GNRHR, since this gene causes isolated hypogonadotrophic hypogonadism (but not Kallman syndrome).
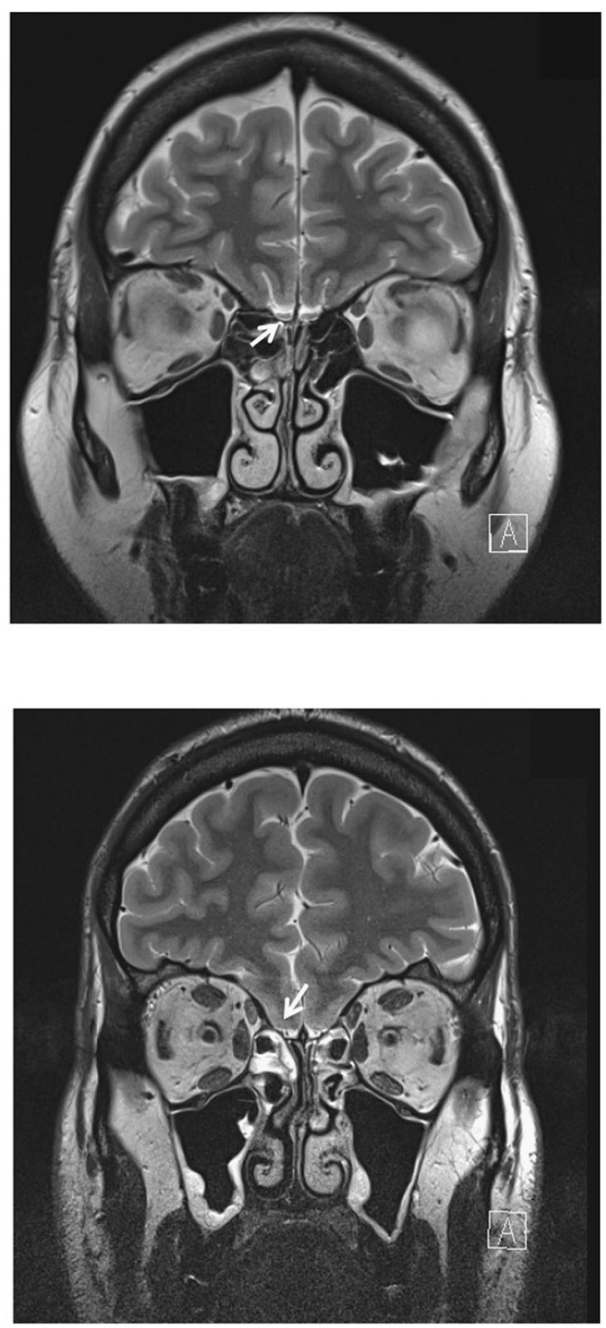

coronal images that were acquired with a high resolution of $0.4 \times 0.4$ $\mathrm{mm}$. The left panel shows a sagittal reconstruction that has a lower inplan resolution $(1.6 \times 0.4 \mathrm{~mm})$ 
Table 2 Bilateral olfactory bulb volume (mm3) and olfactory sulcus depth (mm) among normosmics (a) and anosmics (b)

\begin{tabular}{lllll}
\hline & Rt. Bulb & Lt. Bulb & Rt. Sulcus & Lt. Sulcus \\
\hline a) & & & & \\
N 1 & 80 & 70.4 & 0.59 & 0.79 \\
N 2 & 91.2 & 91.2 & 0.62 & 0.48 \\
N 3 & 91.2 & 88 & 0.96 & 0.7 \\
N 4 & 94.4 & 88 & 0.7 & 0.51 \\
Average & 89.2 & 84.4 & 0.7175 & 0.62 \\
SD & 6.316117 & 9.454452 & 0.168201268 & 0.149443412 \\
b) & & & & \\
A001-1 & 17.6 & 6.4 & No sulcus & No sulcus \\
A001-2 & 20.8 & 12.8 & No sulcus & No sulcus \\
A001-3 & 0 & 0 & No sulcus & No sulcus \\
Average & 12.8 & 6.4 & & \\
SD & 11.2 & 6.4 & & \\
$T$-test & 0.001907 & $4.86 \mathrm{E}-05$ & & \\
\hline Anosmics & & & &
\end{tabular}

Anosmics results confirm their diagnosis

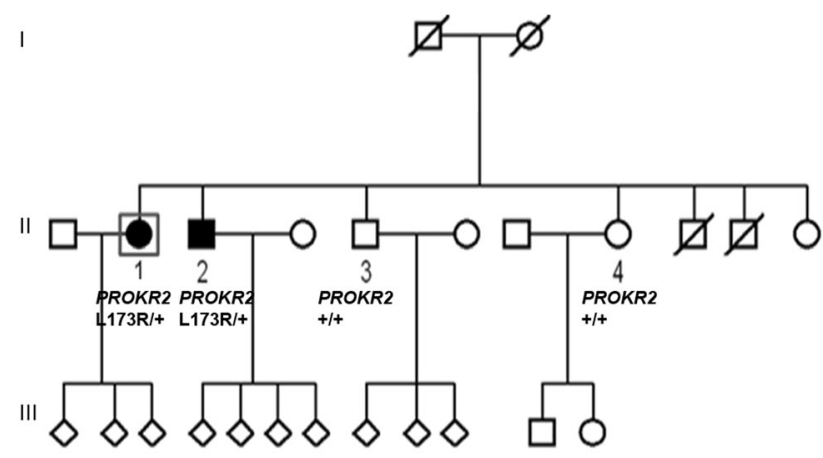

Fig. 4 A055 pedigree with isolated congenital anosmia

\section{Discussion}

The present paper summarizes next generation sequencing (exome, genome, and panel analyses) in 37 ethnically diverse Israeli families with ostensibly isolated congenital anosmia. In eight of these families that were consanguineous and/or multiply affected we subjected 32 individuals to whole exome/genome sequencing. A hypothesis-free approach didn't identify any strong candidate variant in these families. However, enrichment analysis identified three different variants in genes previously implicated in Kallmann syndrome. Further, to validate these results, targeted sequencing was performed on 29 additional families, using a Kallmann syndrome/hypogonadotrophic hypogonadism available diagnostic gene panel of 16 genes. This resulted in the identification of four yet unreported rare variants (frequency of $0-0.0002$ ) in four Kallmann syndrome genes, SEMA3A, CHD7, PROK2 and ANOS1. In three other cases we identified variants already reported for Kallmann syndrome or hypogonadotrophic hypogonadism (PROKR2, SEMA3A, GNRHR).

Three variants with probable pathogenic effect in Table 1 have minor allele frequencies between 0.2 and $1.4 \%$ in the EXAC population, which is higher frequency than expected from variants involved in rare condition such as isolated congenital anosmia with a frequency estimated as $1 / 10,000$ [2]. This suggests that at least some of these variants have incomplete penetrance and/or are involved in oligogenic mode of inheritance. The functional effect of the other variants with unknown significance listed in Table 1 should be investigated in the future, in order to better understand the pathophysiology of anosmia, as well as to reduce likelihood of spurious results in our study.

The fact that one of the variant was found in GNRHR gene is surprising, since homozygous and compound heterozygous variants within this gene were found to cause isolated hypogonadotrophic hypogonadism [16]. However, since the variant in our patient is in a heterozygous state, this individual is not expected to have isolated hypogonadotrophic hypogonadism. In all the families except A227, including 6 consanguineous (Table 1), the identified variation was heterozygous, and the likely inheritance mode was dominant or dominant with partial penetrance. This mode of inheritance resembles a complex mode of inheritance, reported for Kallmann syndrome [46]. Overall, the results shown in Table 1 provide considerable support to the notion that what seems to be an isolated congenital anosmia can result from variants in genes hitherto known to underlie Kallmann syndrome.

These findings lend support to a central message of the present paper: that non-syndromic anosmia could share underlying genetic architecture with Kallmann syndrome, or even with some forms of hypogonadotrophic hypogonadism. A similar conclusion, regarding Kallman syndrome, has been reached previously [10], whereby 5 Kallmann syndrome genes underwent targeted sequencing in 25 isolated congenital anosmia subjects, leading to the identification of variations in the PROKR2 and PROK2 genes in 4 subjects. In our study we found candidate variants in these two genes and in additional four Kallmann syndrome genes. The current study considerably strengthens the conclusions, as it included hypothesis-free exome sequencing and a larger panel of genes, as opposed to hypothesis-dependent five gene panel sequencing.

In addition, our study also suggests for the first time that ostensibly isolated congenital anosmia can be transmitted in di-genic manner. In one of the sequenced families (A001) two different Kallmann genes (FGFR1 and SEMA3A) were found to have heterozygous rare variants. Each of these variants alone displayed a dominant inheritance with 
incomplete penetrance, i.e., could not underlie the disease via a simple Mendelian model. This led to the suggestion of di-genic inheritance, which was further supported by our segregation analysis. A similar di-genic mode of inheritance was suggested for Kallmann syndrome [17]. One of these oligogenic cases [36] even involve the same exact two genes (FGFRI and SEMA3A) found by us, and the authors assert that the SEMA3A variant alone is not sufficient to induce Kallmann syndrome, but contributes to the FGFRI pathogenesis [36]. Taken together, the genetic heterogeneity and modes of inheritance of this trait are more complicated than previously described.

A major limitation of this study is the fact that isolated anosmia diagnosis (based on smell functioning test and patient self-report) cannot exclude the possibility that some patients had minor hypogonadotrophic hypogonadism clinic, which they were not aware of or did not report at recruitment. Despite examination, we did not perform blood hormonal profile measurements, to further exclude this possibility. Unfortunately, this is beyond the scope of the current study.

The fact that no novel candidate genes were found in this sequencing effort represents a major challenge of rare variant studies in complex diseases. The small number of exome sequenced patients (8 unrelated patients) doesn't allow us to perform rare variant gene-based burden testing, since larger sample sizes are needed to perform fully powered case-control study [31]. The small sample size limitation, combined with a suspected non Mendelian mode of inheritance, may lead to a significant risk for false positive results. This potential risk is even complicated by the fact that our variants are mostly missense ones, with unknown significance. Therefore, larger scale exome/genome sequencing studies in well phenotyped patients are needed to resolve numerous isolated congenital anosmia families in our and others arsenal.

The reported FGFR1 p.(R250W) variant in A001 family was previously identified in two unrelated cases with familial and sporadic Kallmann syndrome [37]. Both cases were shown to have abnormal olfactory bulb development similar to our patients with ostensibly pure anosmia [37]. The same variant was also found in five Chinese relatives, four of them affected by Kallmann syndrome and another one carried the same variant but had a normal phenotype, similarly to patient II-1 in family A001. [38]. Interestingly, the same variant was also found in sporadic case with Kallmann syndrome, cleft palate, face dysmorphia and atrial septal defect [39] highlighting the phenotype heterogeneity in patients carrying this p.R250W variant in FGFRI gene.

The $C H D 7$ p.L2806V variant was previously reported in patients referred to diagnostic laboratory for confirmation of the clinical diagnosis of CHARGE syndrome [40, 41], but it was classified as a polymorphism since it was found in one of the parents $[40,41]$ (Table 1). Currently, no information is available regarding the involvement of this variant in Kallmann syndrome. Our finding is consistent with the fact that some variants in $C H D 7$ were previously reported to cause Kallmann syndrome but not the full CHARGE syndrome [47].

In general, genetic variations in genes mediating odorant signal transduction, sensory neuronal development and higher neuronal processing, could constitute the genetic basis for isolated congenital anosmia [29]. A role for olfactory transduction genes in isolated anosmia has received early support via mouse gene deletion experiments [48-50]. However, an early study from our own group failed to identify candidate variants in all three transduction genes by screening of exonic variants in 41-64 unrelated isolated congenital anosmia subjects [2]. Our current nextgeneration sequencing study didn't identify rare strong candidate variants in genes related directly to olfactory transduction either. In another study [8], a loss of function variation in $C N G A 2$ gene was found in only one of 31 unrelated isolated congenital anosmia individuals. Thus a transduction gene with strong a-priori probability to be causative for isolated congenital anosmia is represented (at least until now) only in a small minority of the cases of isolated congenital anosmia.

Finally, the present report represents one of the first large scale next generation sequencing efforts to identify congenital anosmia genes. It argues that there is an overlap in genetic architecture of isolated anosmia and Kallmann syndrome. More generally, isolated congenital anosmia could serve as a platform to better understanding other oligogenic traits and diseases.

Acknowledgements This research was supported by Nella and Leon Benoziyo Center for Neurological Diseases, Weizmann Institute of Science; The Crown Human Genome Center, Weizmann Institute of Science; and by The Legacy Heritage Biomedical Science Partnership Program of the Israel Science Foundation, 586/11.

\section{Compliance with ethical standards}

Conflict of interests The authors declare that they have no competing interests.

\section{References}

1. Karstensen HG, Tommerup N. Isolated and syndromic forms of congenital anosmia. Clin Genet. 2012;81:210-5.

2. Feldmesser E, Bercovich D, Avidan N, et al. Mutations in olfactory signal transduction genes are not a major cause of human congenital general anosmia. Chem Sense. 2007;32:21-30.

3. Dode C, Teixeira L, Levilliers J, et al. Kallmann syndrome: mutations in the genes encoding prokineticin- 2 and prokineticin receptor-2. PLoS Genet. 2006;2:e175. 
4. Weiss J, Pyrski M, Jacobi E, et al. Loss-of-function mutations in sodium channel Nav1.7 cause anosmia. Nature. 2011;472:186-90.

5. Kulaga HM, Leitch CC, Eichers ER, et al. Loss of BBS proteins causes anosmia in humans and defects in olfactory cilia structure and function in the mouse. Nat Genet. 2004;36:994-98.

6. McEwen DP, Koenekoop RK, Khanna H, et al. Hypomorphic CEP290/NPHP6 mutations result in anosmia caused by the selective loss of $\mathrm{G}$ proteins in cilia of olfactory sensory neurons. Proc Natl Acad Sci U S A. 2007;104:15917-922.

7. Skjeldal OH, Stokke O, Refsum S, Norseth J, Petit H. Clinical and biochemical heterogeneity in conditions with phytanic acid accumulation. J Neurol Sci. 1987;77:87-96.

8. Karstensen HG, Mang Y, Fark T, Hummel T, Tommerup N. The first mutation in CNGA2 in two brothers with anosmia. Clin Genet. 2015;88:293-96.

9. Ghadami M, Morovvati S, Majidzadeh AK, et al. Isolated congenital anosmia locus maps to 18p11.23-q12.2. J Med Genet. 2004;41:299-303.

10. Moya-Plana A, Villanueva C, Laccourreye O, Bonfils P, de Roux $\mathrm{N}$. PROKR2 and PROK2 mutations cause isolated congenital anosmia without gonadotropic deficiency. Eur $\mathrm{J}$ Endocrinol. 2013;168:31-37.

11. Alkelai A, Olender T, Haffner-Krausz R et al. A role for TENM1 mutations in congenital general anosmia. Clin Genet. 2016;90:211-19.

12. Mitchell AL, Dwyer A, Pitteloud N, Quinton R. Genetic basis and variable phenotypic expression of Kallmann syndrome: towards a unifying theory. Trend Endocrinol Metab. 2011;22:249-58.

13. Hipkin LJ, Casson IF, Davis JC. Identical twins discordant for Kallmann's syndrome. J Med Genet. 1990;27:198-99.

14. Pitteloud N, Quinton R, Pearce S, et al. Digenic mutations account for variable phenotypes in idiopathic hypogonadotropic hypogonadism. J Clin Invest. 2007;117:457-63.

15. Pitteloud N, Meysing A, Quinton R, et al. Mutations in fibroblast growth factor receptor 1 cause Kallmann syndrome with a wide spectrum of reproductive phenotypes. Mol Cell Endocrinol. 2006;254-255:60-69.

16. Kim SH: Congenital hypogonadotropic hypogonadism and kallmann syndrome: past, present, and future. Endocrinol Metab 2015; 30: 456-66.

17. Boehm U, Bouloux PM, Dattani MT, et al. Expert consensus document: European Consensus Statement on congenital hypogonadotropic hypogonadism--pathogenesis, diagnosis and treatment. Nat Rev Endocrinol. 2015;11:547-64.

18. Quaynor SD, Kim HG, Cappello EM, et al. The prevalence of digenic mutations in patients with normosmic hypogonadotropic hypogonadism and Kallmann syndrome. Fertil Steril. 2011;96:1424-30 e1426.

19. Dode C, Hardelin JP. Kallmann syndrome. Eur J Hum Genet. 2009;17:139-46.

20. Li H, Durbin R. Fast and accurate short read alignment with burrows-wheeler transform. Bioinformatics. 2009;25:1754-760.

21. Li H, Handsaker B, Wysoker A, et al. The sequence alignment/ map format and SAMtools. Bioinformatics. 2009;25:2078-79.

22. McKenna A, Hanna M, Banks E, et al. The genome analysis toolkit: a MapReduce framework for analyzing next-generation DNA sequencing data. Genome Res. 2010;20:1297-1303.

23. Wang K, Li M, Hakonarson H. ANNOVAR: functional annotation of genetic variants from high-throughput sequencing data. Nucleic Acid Res. 2010;38:e164.

24. Liu X, Jian X, Boerwinkle E. dbNSFPv2.0: a database of human non-synonymous SNVs and their functional predictions and annotations. Hum Mutat. 2013;34:E2393-2402.
25. Zhu M, Need AC, Han Y, et al. Using ERDS to infer copynumber variants in high-coverage genomes. Am J Hum Genet. 2012;91:408-21.

26. Krumm N, Sudmant $\mathrm{PH}$, Ko A, et al. Copy number variation detection and genotyping from exome sequence data. Genome Res. 2012;22:1525-32.

27. Purcell S, Neale B, Todd-Brown K, et al. PLINK: a tool set for whole-genome association and population-based linkage analyses. Am J Hum Genet. 2007;81:559-75.

28. Manichaikul A, Mychaleckyj JC, Rich SS, Daly K, Sale M, Chen WM. Robust relationship inference in genome-wide association studies. Bioinformatics. 2010;26:2867-73.

29. Keydar I, Ben-Asher E, Feldmesser E, et al. General olfactory sensitivity database (GOSdb): candidate genes and their genomic variations. Hum Mutat. 2013;34:32-41.

30. Stelzer G, Plaschkes I, Oz-Levi D et al. VarElect: the phenotypebased variation prioritizer of the GeneCards Suite. BMC Genomics 2016; In Press.

31. Goes FS, Pirooznia M, Parla JS, et al. Exome sequencing of familial bipolar disorder. JAMA Psychiatr. 2016;73:590-97.

32. Petrovski S, Wang Q, Heinzen EL, Allen AS, Goldstein DB. Genic intolerance to functional variation and the interpretation of personal genomes. PLoS Genet. 2013;9:e1003709.

33. Rombaux P, Grandin C, Duprez T. How to measure olfactory bulb volume and olfactory sulcus depth? B-ENT. 2009;5:53-60.

34. Duprez TP, Rombaux P. Imaging the olfactory tract (cranial nerve \#1). Eur J Radiol. 2010;74:288-98.

35. Marcos S, Sarfati J, Leroy C, et al. The prevalence of CHD7 missense versus truncating mutations is higher in patients with Kallmann syndrome than in typical CHARGE patients. J Clin Endocrinol Metab. 2014;99:E2138-43.

36. Hanchate NK, Giacobini P, Lhuillier P, et al. SEMA3A, a gene involved in axonal pathfinding, is mutated in patients with Kallmann syndrome. PLoS Genet. 2012;8:e1002896.

37. Trarbach EB, Costa EM, Versiani B, et al. Novel fibroblast growth factor receptor 1 mutations in patients with congenital hypogonadotropic hypogonadism with and without anosmia. J Clin Endocrinol Metab. 2006;91:4006-12.

38. Gu WJ, Zhang Q, Wang YQ, et al. Mutation analyses in pedigrees and sporadic cases of ethnic Han Chinese Kallmann syndrome patients. Exp Biol Med. 2015;240:1480-89.

39. Dode C, Fouveaut C, Mortier G, et al. Novel FGFR1 sequence variants in Kallmann syndrome, and genetic evidence that the FGFR1c isoform is required in olfactory bulb and palate morphogenesis. Human Mutat. 2007;28:97-98.

40. Vuorela P, Ala-Mello S, Saloranta C, et al. Molecular analysis of the CHD7 gene in CHARGE syndrome: identification of 22 novel mutations and evidence for a low contribution of large CHD7 deletions. Genet Med. 2007;9:690-94.

41. Bartels CF, Scacheri C, White L, Scacheri PC, Bale S. Mutations in the CHD7 gene: the experience of a commercial laboratory. Genet Test Mol Biomark. 2010;14:881-91.

42. Dode C, Levilliers J, Dupont JM, et al. Loss-of-function mutations in FGFR1 cause autosomal dominant Kallmann syndrome. Nat Genet. 2003;33:463-65.

43. Young J, Metay C, Bouligand J, et al. SEMA3A deletion in a family with Kallmann syndrome validates the role of semaphorin $3 \mathrm{~A}$ in human puberty and olfactory system development. Hum Reprod. 2012;27:1460-65.

44. Waldstreicher J, Seminara SB, Jameson JL, et al. The genetic and clinical heterogeneity of gonadotropin-releasing hormone deficiency in the human. J Clin Endocrinol Metab. 1996;81:4388-95.

45. Layman LC. Clinical genetic testing for Kallmann syndrome. J Clin Endocrinol Metab. 2013;98:1860-62. 
46. Hardelin JP, Dode C. The complex genetics of Kallmann syndrome: KAL1, FGFR1, FGF8, PROKR2, PROK2, et al. Sex Dev. 2008;2:181-93.

47. Jongmans MC, van Ravenswaaij-Arts CM, Pitteloud N, et al. CHD7 mutations in patients initially diagnosed with Kallmann syndrome--the clinical overlap with CHARGE syndrome. Clin Genet. 2009;75:65-71.
48. Belluscio L, Gold GH, Nemes A, Axel R. Mice deficient in G(olf) are anosmic. Neuron. 1998;20:69-81.

49. Wong ST, Trinh K, Hacker B, et al. Disruption of the type III adenylyl cyclase gene leads to peripheral and behavioral anosmia in transgenic mice. Neuron. 2000;27:487-97.

50. Brunet LJ, Gold GH, Ngai J. General anosmia caused by a targeted disruption of the mouse olfactory cyclic nucleotide-gated cation channel. Neuron. 1996;17:681-93. 